\title{
Efficacy and Safety of Incontinence Surgery According to the Surgeon's Specialty and Performance of a Preoperative Urodynamic Study
}

\author{
Jin Bong Choi ${ }^{1}$, Kyung-Do Han ${ }^{2}$, U-Syn Ha ${ }^{3}$, Sung-Hoo Hong ${ }^{3}$ \\ ${ }^{1}$ Department of Urology, Bucheon St. Mary's Hospital, College of Medicine, The Catholic University of Korea, Bucheon, Korea \\ ${ }^{2}$ Department of Biostatistics, College of Medicine, The Catholic University of Korea, Seoul, Korea \\ ${ }^{3}$ Department of Urology, Seoul St. Mary's Hospital, College of Medicine, The Catholic University of Korea, Seoul, Korea
}

Purpose: The aim of this study was to analyze the efficacy and to estimate the complication rate of incontinence surgery according to the surgeon's specialty and whether a preoperative urodynamic study (UDS) was performed, using a nationally representative dataset.

Methods: We enrolled 356,155 women over 20 years old who had undergone surgery for stress urinary incontinence between 2006 and 2015. Patients were followed for up to 3 years to analyze the reoperation and complication rates. Data were obtained from the National Health Claims Database of the National Health Insurance Service (NHIS) of Korea. Multiple Cox regression analysis was conducted to examine the efficacy and safety of incontinence surgery according to the surgeon's specialty and whether a preoperative UDS was performed.

Results: The hazard ratio (HR) for reoperation was significantly higher for procedures performed by nonurologists than for procedures performed by urologists (HR, 1.174; 95\% confidence interval [CI], 1.103-1.249). Acute urinary retention, postoperative infections, procedure-associated pain, and other complications were also more common in procedures performed by nonurologists than in those performed by urologists. When stratified by whether a preoperative UDS was performed, the HR for reoperation according to the surgeon's specialty varied by performance of a preoperative UDS. While the reoperation rate was significantly higher in procedures performed by non-urologists when a preoperative UDS was performed (HR, 1.208; 95\% CI, 1.122-1.3), there was no significant difference in the HRs for reoperation according to specialty when a preoperative UDS was not performed.

Conclusions: This population-based study showed that the postoperative outcomes of incontinence surgery were dependent upon the surgeon's specialty and that the reoperation rate according to the surgeon's specialty varied based on whether a preoperative UDS was performed.

Keywords: Stress urinary incontinence; Surgery; Outcomes; Urodynamics

- Grant/Fund Support: This study was supported by the Foundation of the Korean Urological Association in 2017 (FKUA-2017-001).

- Research Ethics: This study was approved by the Institutional Review Board of the Catholic University of Korea (KC17EESI0443).

- Conflict of Interest: No potential conflict of interest relevant to this article was reported.

Corresponding author: Sung-Hoo Hong (iD https://orcid.org/0000-0001-7432-4209 Department of Urology, Seoul St. Mary's Hospital, College of Medicine, The Catholic University of Korea, 222 Banpo-daero, Seocho-gu, Seoul 06591, Korea E-mail: toomey@catholic.ac.kr / Tel: +82-2-2258-6229 / Fax: +82-2-2258-1536 Submitted: May 2, 2018 / Accepted after revision: June 21, 2018 (i) (5) This is an Open Access article distributed under the terms of the Creative Commons Attribution Non-Commercial License (http://creativecommons.org/licenses/by-nc/4.0/) which permits unrestricted non-commercial use, distribution, and reproduction in any medium, provided the original work is properly cited. 


\section{INTRODUCTION}

Stress urinary incontinence (SUI) is defined as the involuntary loss of urine during physical exertion or when sneezing or coughing [1]. SUI can diminish patients' quality of life and imposes an increased economic burden $[2,3]$. The prevalence of urinary incontinence varies according to the study population and inclusion criteria, but it has been reported to be as high as $49 \%$, and it increases with age [4]. Approximately $50 \%$ of patients with urinary incontinence complain of SUI symptoms [5].

Both nonsurgical and surgical options can be used to treat SUI. Weight loss and exercise to strengthen pelvic-floor muscles, such as Kegel exercises, can improve SUI symptoms [6,7]. Electrostimulation may also strengthen pelvic-floor muscles, but there is insufficient evidence of its efficacy. Furthermore, there are no U.S. Food and Drug Administration-approved medications specifically for SUI [8]. Thus, physicians usually suggest surgery to improve symptoms when other treatments are unsuccessful.

In the United States, the annual number of urinary incontinence operations increased from 37,953 in 1998 to 94,910 in 2007 , concomitantly with the introduction of minimally invasive operative techniques. By 2007, midurethral sling surgery increased to $75.2 \%$ of incontinency operations, while the retropubic suspension technique decreased to $13.8 \%$ [9]. In Korea, the proportion of transvaginal operations that used a midurethral sling also continued to increase [10]. Consequently, postoperative complications associated with incontinence surgery have also been increasing. Postoperative complications can have a devastating effect on individual patients and pose substantial additional cost burdens to the health care system.

Therefore, in order to reduce postoperative complications, it is important to identify preoperative clinical risk factors and to improve intraoperative procedures [11], which requires high levels of physician expertise. To this end, the aim of this study was to analyze the efficacy and to estimate the complication rate of incontinence surgery according to the surgeon's specialty using a nationally representative dataset. In addition, we also examined the impact of performing a preoperative urodynamic study (UDS) on the efficacy and safety of incontinence surgery according to the surgeon's specialty.

\section{MATERIALS AND METHODS}

\section{Study Population}

This study was approved by the Institutional Review Board of the Catholic University of Korea (KC17EESI0443). The study subjects included 356,155 women over 20 years old who underwent surgery for SUI between 2006 and 2015. Patients were followed for up to 3 years to analyze the reoperation and complication rates. Data were obtained from the National Health Claims Database of the National Health Insurance Service (NHIS) of Korea. This database has been described previously in a prior population-based study [12].

In the NHIS system, surgeons must enter a specific code for the operation performed to bill the correct insurance cost. For our analyses, incontinence surgery included transvaginal operations using a midurethral sling procedure (R3560, R3561, R3564, and R3565), an abdominal-approach procedure (R3562), and a foreign material or autologous fat-injection procedure (R3563). Preoperative UDS was coded as E6560, E6561, E6563, E6564, or EZ752. For all procedures, the surgeon's specialty was identifiable.

Data on age, income, and year of operation were collected. We also searched insurance claim codes based on the International Classification of Diseases, 10th Revision, Clinical Modifications to define other confounding factors and outcome variables. SUI was coded as N393, N394, or R32. The inclusion criteria were patients with both a SUI code and an incontinence surgery code. Hypertension, diabetes, and dyslipidemia were identified according to methods described in a previous study [12].

\section{Outcome Variables}

We included several categories of complications that occurred within 3 months after surgery. Postoperative infections included cystitis (N30), tubulointerstitial nephritis (N10, N11, and N12), obstructive and reflux uropathy (N13), renal tubulointerstitial disorders (N16), urethritis and urethral syndrome (N34), fever due to infections of unknown origin (R50), shock (R57), urinary tract infection (N39), other female pelvic inflammatory diseases (N73), other inflammation of the vagina and vulva (N76), and vulvovaginal ulceration and inflammation (N77). Procedure-associated pain included dyspareunia (N941), abdominal and pelvic pain (R10), and pain associated with micturition (R30). Other complications included unspecified symptoms involving the genitourinary system (R39), procedural complications not elsewhere classified (T81), complications involving genitourinary prosthetic devices, implants, or grafts (T83), urethral stricture (N35), urethral fistula (N36), and urethral disorders from diseases classified elsewhere (N37).

Postoperative acute urinary retention was defined as the addition of any of the following procedure codes within 1 month 
after surgery: Nelaton catheterization (M0050) or Foley catheterization (M0060). A bleeding complication was defined as the addition of any of the following blood codes within 1 month after surgery: X2021, X2022, X2031, X2032, X2091, X2092, X2111, X2112, X2131, or X2132. Lastly, the efficacy of incontinence surgery was assessed based on whether a patient underwent reoperation within 3 years.

\section{Statistical Analysis}

We used SAS ver. 9.4 (SAS Institute Inc., Cary, NC, USA) for all analyses. Data are presented as mean \pm standard deviation values for continuous variables and as proportions for categorical variables. Multiple Cox regression analysis was conducted to estimate hazard ratios (HRs) and 95\% confidence interval (CIs) for the efficacy and safety of incontinence surgery according to the surgeon's specialty and whether a preoperative UDS was performed. We adjusted for age, income, hypertension, diabetes, and dyslipidemia in our models. Values of $\mathrm{P}<0.05$ were considered to indicate statistical significance.

\section{RESULTS}

\section{Trends in the Total Number of Patients Who Underwent Incontinence Surgery}

The number of patients who underwent surgery for SUI decreased continuously from $2006(n=60,520)$ to $2015(n=24,599)$ (Table 1). When we compared the number of incontinence operations performed by specialty, $40 \%$ were performed by urologists, and the other $60 \%$ were performed by nonurologists, indi-

Table 1. Trends in the total number of patients who underwent incontinence surgery per year

\begin{tabular}{lccc}
\hline Year & Urology & Others & Total (n) \\
\hline 2006 & $23,524(38.87)$ & $36,996(61.13)$ & 60,520 \\
2007 & $18,371(40.49)$ & $26,997(59.51)$ & 45,368 \\
2008 & $16,360(40.43)$ & $24,101(59.57)$ & 40,461 \\
2009 & $16,098(40.09)$ & $24,052(59.91)$ & 40,150 \\
2010 & $12,685(38.99)$ & $19,853(61.01)$ & 32,538 \\
2011 & $12,224(39.37)$ & $18,828(60.63)$ & 31,052 \\
2012 & $10,872(38.64)$ & $17,265(61.36)$ & 28,137 \\
2013 & $10,590(39.41)$ & $16,283(60.59)$ & 26,873 \\
2014 & $10,386(39.26)$ & $16,071(60.74)$ & 26,457 \\
2015 & $9,475(38.52)$ & $15,124(61.48)$ & 24,599 \\
\hline
\end{tabular}

Values are presented as number (\%).
Table 2. Baseline characteristics and clinical outcomes according to surgeon specialty

\begin{tabular}{|c|c|c|c|}
\hline Variable & Urologist & Nonurologist & Total \\
\hline \multicolumn{4}{|l|}{ Age (yr) } \\
\hline $20-29$ & $347(0.25)$ & $741(0.34)$ & $1,088(0.31)$ \\
\hline $30-39$ & $13,468(9.58)$ & $27,428(12.72)$ & $40,896(11.48)$ \\
\hline $40-49$ & $54,683(38.9)$ & $100,412(46.58)$ & $155,095(43.55)$ \\
\hline $50-59$ & $47,753(33.97)$ & $64,500(29.92)$ & $112,253(31.52)$ \\
\hline $60-69$ & $18,024(12.82)$ & $17,819(8.27)$ & $35,843(10.06)$ \\
\hline $70-79$ & $5,758(4.1)$ & $4,326(2.01)$ & $10,084(2.83)$ \\
\hline $80-89$ & $544(0.39)$ & $338(0.16)$ & $882(0.25)$ \\
\hline$\geq 90$ & $8(0.01)$ & $6(0)$ & $14(0)$ \\
\hline \multicolumn{4}{|c|}{ House income $e^{\mathrm{a})}$} \\
\hline Medical aid & $4,194(2.98)$ & $4,522(2.1)$ & $8,716(2.45)$ \\
\hline Q1 & $34,448(24.5)$ & $55,997(25.98)$ & $90,445(25.39)$ \\
\hline Q2 & $29,425(20.93)$ & $48,106(22.32)$ & $77,531(21.77)$ \\
\hline Q3 & $32,066(22.81)$ & $49,145(22.8)$ & $81,211(22.8)$ \\
\hline Q4 & $40,452(28.77)$ & $57,800(26.81)$ & $98,252(27.59)$ \\
\hline \multicolumn{4}{|l|}{ Diabetes } \\
\hline No & $130,451(92.79)$ & $203,886(94.58)$ & $334,337(93.87)$ \\
\hline Yes & $10,134(7.21)$ & $11,684(5.42)$ & $21,818(6.13)$ \\
\hline \multicolumn{4}{|l|}{ Hypertension } \\
\hline No & $109,837(78.13)$ & $177,522(82.35)$ & $287,359(80.68)$ \\
\hline Yes & $30,748(21.87)$ & $38,048(17.65)$ & $68,796(19.32)$ \\
\hline \multicolumn{4}{|l|}{ Dyslipidemia } \\
\hline No & $118,966(84.62)$ & $190,238(88.25)$ & $309,204(86.82)$ \\
\hline Yes & $21,619(15.38)$ & $25,332(11.75)$ & $46,951(13.18)$ \\
\hline \multicolumn{4}{|c|}{ Preoperative UDS } \\
\hline No & $39,122(27.83)$ & $55,740(25.86)$ & $94,862(26.64)$ \\
\hline Yes & $101,463(72.17)$ & $159,830(74.14)$ & $261,293(73.36)$ \\
\hline \multicolumn{4}{|l|}{ Reoperation } \\
\hline No & $138,965(98.85)$ & $212,815(98.72)$ & $351,780(98.77)$ \\
\hline Yes & $1,620(1.15)$ & $2,755(1.28)$ & $4,375(1.23)$ \\
\hline \multicolumn{4}{|l|}{ Transfusion } \\
\hline No & 140,487 (99.93) & $215,462(99.95)$ & 355,949 (99.94) \\
\hline Yes & $98(0.07)$ & $108(0.05)$ & $206(0.06)$ \\
\hline \multicolumn{4}{|l|}{ AUR } \\
\hline No & $137,136(97.55)$ & 205,798 (95.47) & $342,934(96.29)$ \\
\hline Yes & $3,449(2.45)$ & $9,772(4.53)$ & $13,221(3.71)$ \\
\hline \multicolumn{4}{|l|}{ Infection } \\
\hline No & $77,879(55.4)$ & $89,887(41.7)$ & $167,766(47.11)$ \\
\hline Yes & $62,706(44.6)$ & $125,683(58.3)$ & $188,389(52.89)$ \\
\hline \multicolumn{4}{|l|}{ Pain } \\
\hline No & $129,116(91.84)$ & $191,170(88.68)$ & $320,286(89.93)$ \\
\hline Yes & $11,469(8.16)$ & $24,400(11.32)$ & $138,869(10.07)$ \\
\hline \multicolumn{4}{|l|}{ Others } \\
\hline No & $136,732(97.26)$ & 205,837 (95.48) & $342,569(96.46)$ \\
\hline Yes & $3,853(2.74)$ & $9,733(4.52)$ & $13,586(3.54)$ \\
\hline
\end{tabular}

Values are presented as the number (\%).

UDS, urodynamic study; AUR, acute urinary retention.

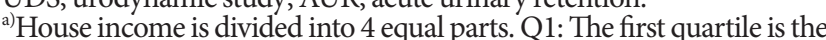
income below which lies the $25 \%$ of the bottom data. Q2: The second quartile divides the range in the middle and has $50 \%$ of the data below it. Q3: The third quartile has 75\% of the data below it. Q4: The top 25\% of the data above it. 
Table 3. Efficacy and safety of incontinence surgery according to surgeon specialty

\begin{tabular}{|c|c|c|c|c|c|c|c|}
\hline \multirow{2}{*}{ Parameter \& specialty } & \multirow{2}{*}{ Total } & \multirow{2}{*}{ Event } & \multirow{2}{*}{ Person-years } & \multirow{2}{*}{ Incidence } & \multicolumn{3}{|c|}{ HR (95\% confidence interval) } \\
\hline & & & & & Model 1 ${ }^{\text {a) }}$ & Model $2^{\text {b) }}$ & Model $3^{\text {c) }}$ \\
\hline \multicolumn{8}{|l|}{ Reoperation } \\
\hline Urologist & 140,585 & 1,620 & $145,834,347$ & 0.011 & Reference & Reference & Reference \\
\hline Nonurologist & 215,570 & 2,755 & $223,266,339$ & 0.012 & $1.111(1.044-1.181)$ & $1.174(1.104-1.249)$ & $1.174(1.103-1.249)$ \\
\hline \multicolumn{8}{|l|}{ Transfusion } \\
\hline Urologist & 140,585 & 98 & $4,215,980$ & 0.023 & Reference & Reference & Reference \\
\hline Nonurologist & 215,570 & 108 & $6,465,115$ & 0.017 & $0.719(0.547-0.945)$ & $0.798(0.605-1.052)$ & $0.806(0.611-1.064)$ \\
\hline \multicolumn{8}{|l|}{ AUR } \\
\hline Urologist & 140,585 & 3,449 & $4,148,294$ & 0.831 & Reference & Reference & Reference \\
\hline Nonurologist & 215,570 & 9,772 & $6,313,566$ & 1.548 & $1.858(1.787-1.931)$ & $1.92(1.846-1.997)$ & $1.923(1.849-1.999)$ \\
\hline \multicolumn{8}{|l|}{ Infection } \\
\hline Urologist & 140,585 & 62,706 & $8,079,187$ & 7.761 & Reference & Reference & Reference \\
\hline Nonurologist & 215,570 & 125,683 & $9,962,593$ & 12.616 & $1.476(1.462-1.49)$ & $1.446(1.432-1.46)$ & $1.446(1.433-1.46)$ \\
\hline \multicolumn{8}{|l|}{ Pain } \\
\hline Urologist & 140,585 & 11,469 & $11,918,399$ & 0.962 & Reference & Reference & Reference \\
\hline Nonurologist & 215,570 & 24,400 & $17,693,956$ & 1.379 & $1.420(1.389-1.452)$ & $1.426(1.394-1.458)$ & $1.428(1.396-1.46)$ \\
\hline \multicolumn{8}{|l|}{ Others } \\
\hline Urologist & 140,585 & 3,853 & $12,382,034$ & 0.311 & Reference & Reference & Reference \\
\hline Nonurologist & 215,570 & 9,733 & $18,659,187$ & 0.521 & $1.668(1.607-1.731)$ & $1.707(1.644-1.773)$ & $1.706(1.643-1.772)$ \\
\hline
\end{tabular}

All rates are expressed as number per 1,000 person-years.

AUR, acute urinary retention; $\mathrm{HR}$, hazard ratio.

a) Unadjusted. ${ }^{\text {b) }}$ Adjusted for age. ${ }^{\text {c) }}$ Adjusted for age, income, diabetes, hypertension, and dyslipidemia.

cating that the majority of operations were performed in nonurology departments. The ratio of the number of operations according to specialty has remained fairly constant over the last 10 years. When we analyzed surgery patterns according to 10 -year age groups, incontinence surgery was performed most frequently among women in their 40 s, followed by women in their 50 s. The baseline characteristics and clinical outcomes of patients according to the surgeon's specialty are summarized in Table 2.

\section{Efficacy and Safety of Incontinence Surgery According to the Surgeon's Specialty}

Estimates from multiple Cox regression models for the efficacy and safety of incontinence surgery according to the surgeon's specialty are presented in Table 3 . The HR for reoperation was significantly higher in procedures performed by non-urologists (HR, 1.174; 95\% CI, 1.103-1.249) in the multivariable-adjusted model. Acute urinary retention within 1 month occurred more frequently in women who underwent surgery performed by a nonurologist than in those treated by a urologist (HR, 1.923; 95\% CI, 1.849-1.999). Postoperative infections, procedure-associated pain, and other complications were also more common in procedures performed by nonurologists than in proce- dures performed by urologists (HR, 1.446; 95\% CI, 1.433-1.46; HR, 1.428; 95\% CI, 1.396-1.46; and HR, 1.706; 95\% CI, 1.643 1.72 , respectively). The transfusion rate was higher in procedures performed by urologists, but the difference was not statistically significant.

\section{Impact of Preoperative UDS on the Efficacy and Safety of Incontinence Surgery According to the Surgeon's Specialty}

The percentage of incontinence operations not preceded by UDS was $\sim 25 \%$ in both groups. The Ministry of Health and Welfare of the Republic of Korea has recommended preoperative UDS since February 2007 (Notice 2007-3). Therefore, most of the cases without a preoperative UDS in this cohort occurred in 2006 and early 2007.

The results of the multiple Cox regression analysis of the efficacy and safety of incontinence surgery according to the surgeon's specialty when stratified by preoperative UDS implementation are presented in Table 4 . When stratified by performance of a preoperative UDS, the HRs for acute urinary retention, postoperative infection, procedure-associated pain, and other complications were higher in the procedures performed by nonurologists. However, the HRs for reoperation according 
Table 4. Efficacy and safety of incontinence surgery according to surgeon specialty when stratified by preoperative urodynamic study (UDS)

\begin{tabular}{|c|c|c|c|c|c|c|c|}
\hline \multirow{2}{*}{ Parameter \& specialty } & \multirow{2}{*}{ Total } & \multirow{2}{*}{ Event } & \multirow{2}{*}{ Person-years } & \multirow{2}{*}{ Incidence } & \multicolumn{3}{|c|}{ HR ( $95 \%$ confidence interval) } \\
\hline & & & & & Model $1^{\text {a) }}$ & Model 2 ${ }^{\text {b) }}$ & Model $3^{\text {c) }}$ \\
\hline Preoperative UDS, yes & & & & & & & \\
\hline $\begin{array}{l}\text { Reoperation } \\
\text { Urologist } \\
\text { Nonurologist }\end{array}$ & $\begin{array}{l}101,463 \\
159,830\end{array}$ & $\begin{array}{l}1,134 \\
2,014\end{array}$ & $\begin{array}{l}104,688,906 \\
164,384,560\end{array}$ & $\begin{array}{l}0.011 \\
0.012\end{array}$ & $\begin{array}{c}\text { Reference } \\
1.13(1.051-1.216)\end{array}$ & $\begin{array}{c}\text { Reference } \\
1.207(1.122-1.299)\end{array}$ & $\begin{array}{c}\text { Reference } \\
1.208(1.122-1.3)\end{array}$ \\
\hline $\begin{array}{l}\text { Transfusion } \\
\text { Urologist } \\
\text { Nonurologist }\end{array}$ & $\begin{array}{l}101,463 \\
159,830\end{array}$ & $\begin{array}{l}68 \\
75\end{array}$ & $\begin{array}{l}3,042,821 \\
4,793,533\end{array}$ & $\begin{array}{l}0.022 \\
0.016\end{array}$ & $\begin{array}{c}\text { Reference } \\
0.7(0.504-0.972)\end{array}$ & $\begin{array}{c}\text { Reference } \\
0.774(0.555-1.079)\end{array}$ & $\begin{array}{c}\text { Reference } \\
0.782(0.56-1.091)\end{array}$ \\
\hline $\begin{array}{l}\text { AUR } \\
\text { Urologist } \\
\text { Nonurologist }\end{array}$ & $\begin{array}{l}101,463 \\
159,830\end{array}$ & $\begin{array}{l}2,603 \\
7,624\end{array}$ & $\begin{array}{l}2,990,268 \\
4,681,667\end{array}$ & $\begin{array}{l}0.87 \\
1.628\end{array}$ & $\begin{array}{c}\text { Reference } \\
1.868(1.786-1.953)\end{array}$ & $\begin{array}{c}\text { Reference } \\
1.941(1.856-2.03)\end{array}$ & $\begin{array}{c}\text { Reference } \\
1.943(1.858-2.032)\end{array}$ \\
\hline $\begin{array}{l}\text { Infection } \\
\text { Urologist } \\
\text { Nonurologist }\end{array}$ & $\begin{array}{l}101,463 \\
159,830\end{array}$ & $\begin{array}{l}46,627 \\
96,266\end{array}$ & $\begin{array}{l}5,696,999 \\
7,096,468\end{array}$ & $\begin{array}{r}8.185 \\
13.565\end{array}$ & $\begin{array}{c}\text { Reference } \\
1.487(1.471-1.504)\end{array}$ & $\begin{array}{c}\text { Reference } \\
1.453(1.436-1.469)\end{array}$ & $\begin{array}{c}\text { Reference } \\
1.453(1.437-1.47)\end{array}$ \\
\hline $\begin{array}{l}\text { Pain } \\
\text { Urologist } \\
\text { Nonurologist }\end{array}$ & $\begin{array}{l}101,463 \\
159,830\end{array}$ & $\begin{array}{r}8,287 \\
19,117\end{array}$ & $\begin{array}{r}8,603,369 \\
13,036,007\end{array}$ & $\begin{array}{l}0.963 \\
1.466\end{array}$ & $\begin{array}{c}\text { Reference } \\
1.505(1.467-1.544)\end{array}$ & $\begin{array}{c}\text { Reference } \\
1.511(1.472-1.55)\end{array}$ & $\begin{array}{c}\text { Reference } \\
1.511(1.472-1.551)\end{array}$ \\
\hline $\begin{array}{l}\text { Others } \\
\text { Urologist } \\
\text { Nonurologist }\end{array}$ & $\begin{array}{l}101,463 \\
159,830\end{array}$ & $\begin{array}{l}2,840 \\
7,658\end{array}$ & $\begin{array}{r}8,930,418 \\
13,797,392\end{array}$ & $\begin{array}{l}0.318 \\
0.555\end{array}$ & $\begin{array}{c}\text { Reference } \\
1.735(1.662-1.812)\end{array}$ & $\begin{array}{c}\text { Reference } \\
1.76(1.685-1.838)\end{array}$ & $\begin{array}{c}\text { Reference } \\
1.759(1.684-1.837)\end{array}$ \\
\hline Preoperative UDS, no & & & & & & & \\
\hline $\begin{array}{l}\text { Reoperation } \\
\text { Urologist } \\
\text { Nonurologist }\end{array}$ & $\begin{array}{l}39,122 \\
55,740\end{array}$ & $\begin{array}{l}486 \\
741\end{array}$ & $\begin{array}{l}41,145,441 \\
58,881,779\end{array}$ & $\begin{array}{l}0.012 \\
0.013\end{array}$ & $\begin{array}{c}\text { Reference } \\
1.065(0.95-1.194)\end{array}$ & $\begin{array}{c}\text { Reference } \\
1.107(0.986-1.242)\end{array}$ & $\begin{array}{c}\text { Reference } \\
1.107(0.987-1.242)\end{array}$ \\
\hline $\begin{array}{l}\text { Transfusion } \\
\text { Urologist } \\
\text { Nonurologist }\end{array}$ & $\begin{array}{l}39,122 \\
55,740\end{array}$ & $\begin{array}{l}30 \\
33\end{array}$ & $\begin{array}{l}1,173,159 \\
1,671,582\end{array}$ & $\begin{array}{l}0.026 \\
0.02\end{array}$ & $\begin{array}{c}\text { Reference } \\
0.772(0.471-1.266)\end{array}$ & $\begin{array}{c}\text { Reference } \\
0.861(0.523-1.417)\end{array}$ & $\begin{array}{c}\text { Reference } \\
0.865(0.526-1.425)\end{array}$ \\
\hline $\begin{array}{l}\text { AUR } \\
\text { Urologist } \\
\text { Nonurologist }\end{array}$ & $\begin{array}{l}39,122 \\
55,740\end{array}$ & $\begin{array}{r}846 \\
2,148\end{array}$ & $\begin{array}{l}1,158,026 \\
1,631,899\end{array}$ & $\begin{array}{l}0.731 \\
1.316\end{array}$ & $\begin{array}{c}\text { Reference } \\
1.797(1.659-1.945)\end{array}$ & $\begin{array}{c}\text { Reference } \\
1.829(1.689-1.981)\end{array}$ & $\begin{array}{c}\text { Reference } \\
1.831(1.69-1.983)\end{array}$ \\
\hline $\begin{array}{l}\text { Infection } \\
\text { Urologist } \\
\text { Nonurologist }\end{array}$ & $\begin{array}{l}39,122 \\
55,740\end{array}$ & $\begin{array}{l}16,079 \\
29,417\end{array}$ & $\begin{array}{l}2,382,188 \\
2,866,125\end{array}$ & $\begin{array}{c}6.75 \\
10.264\end{array}$ & $\begin{array}{c}\text { Reference } \\
1.424(1.397-1.452)\end{array}$ & $\begin{array}{c}\text { Reference } \\
1.396(1.369-1.423)\end{array}$ & $\begin{array}{c}\text { Reference } \\
1.397(1.37-1.424)\end{array}$ \\
\hline $\begin{array}{l}\text { Pain } \\
\text { Urologist } \\
\text { Nonurologist }\end{array}$ & $\begin{array}{l}39,122 \\
55,740\end{array}$ & $\begin{array}{l}3,182 \\
5,283\end{array}$ & $\begin{array}{l}3,315,030 \\
4,657,949\end{array}$ & $\begin{array}{l}0.96 \\
1.134\end{array}$ & $\begin{array}{c}\text { Reference } \\
1.178(1.127-1.231)\end{array}$ & $\begin{array}{c}\text { Reference } \\
1.181(1.13-1.234)\end{array}$ & $\begin{array}{c}\text { Reference } \\
1.183(1.132-1.237)\end{array}$ \\
\hline $\begin{array}{l}\text { Others } \\
\text { Urologist } \\
\text { Nonurologist }\end{array}$ & $\begin{array}{l}39,122 \\
55,740\end{array}$ & $\begin{array}{l}1,013 \\
2,075\end{array}$ & $\begin{array}{l}3,451,616 \\
4,861,795\end{array}$ & $\begin{array}{l}0.293 \\
0.427\end{array}$ & $\begin{array}{c}\text { Reference } \\
1.45(1.345-1.563)\end{array}$ & $\begin{array}{c}\text { Reference } \\
1.511(1.401-1.629)\end{array}$ & $\begin{array}{c}\text { Reference } \\
1.51(1.4-1.628)\end{array}$ \\
\hline
\end{tabular}

All rates are expressed as number per 1,000 person-years.

HR, hazard ratio; AUR, acute urinary retention.

${ }^{a}$ Unadjusted. ${ }^{\text {b) }}$ Adjusted for age. ${ }^{\text {c) }}$ Adjusted for age, income, diabetes, hypertension, and dyslipidemia.

to the surgeon's specialty varied based on whether a preoperative UDS was performed. Although the reoperation rate was significantly higher in the procedures performed by non-urologists when a preoperative UDS was performed (HR, 1.208; 95\%
CI, 1.122-1.3), there was no significant difference in the HRs for reoperation between the 2 groups when a preoperative UDS was not performed (HR, 1.107; 95\% CI, 0.987 -1.242). 


\section{DISCUSSION}

Several studies have evaluated practice patterns in incontinence surgery according to surgeon specialty [13,14], but few have compared the postoperative outcomes of incontinence surgery across specialties, and the results have varied. Anger et al. [15] suggested that patients who underwent surgery for SUI or pelvic organ prolapse (POP) performed by urologists were more likely to develop urinary tract infections and to undergo reoperation than those who underwent surgery performed by gynecologists based on medical claims data in the USA. In contrast, Slopnick et al. [16] suggested that complication rates were higher among gynecologists than urologists using the American College of Surgeons National Surgical Quality Improvement Program database. Furthermore, it is somewhat difficult to analyze the reoperation rate as a clinical outcome, because patients with recurrent SUI sometimes choose different surgeons for the subsequent reoperation. Elliott et al. [17] reported that approximately one-third of patients with recurrent SUI or POP did not notify their primary surgeon when undergoing a reoperation performed by another surgeon. Therefore, to effectively analyze the reoperation rate, population-based studies are necessary.

In this study of data from the NHIS database, which covers the entire Korean population, we found that the postoperative outcomes of incontinence surgery were dependent upon the surgeon's specialty. The HR for reoperation was significantly lower for urologists than for non-urologists. The following reasons may at least partially explain this finding. First, greater experience can improve clinical outcomes [18]. Most incontinence surgery in teaching hospitals in Korea is performed in the urology department. Therefore, urologists may gain more surgical experience during residency. Furthermore, in the case of postoperative complications such as voiding dysfunction, additional tests such as cystoscopy and UDS that are particularly strongly associated with the specialty of urology may be required. Second, several studies have reported that there were differences in the preferred types of incontinence surgery across specialties, along with variation in the complication rate according to the type of surgery. James et al. found that urologists performed more autologous fascial sling procedures than gynecologists [13]. Wu et al. [14] reported that gynecologists performed more Kelly plications, whereas urologists performed more traditional pubovaginal sling procedures.

In addition, we estimated the impact of preoperative UDS on the efficacy and safety of incontinence surgery according to the surgeon's specialty. UDS is the most effective test to check for preoperative clinical risk factors before incontinence surgery. In this study, we demonstrated that personalized information about patient incontinence may help physicians select the most appropriate treatment option. An online survey reported that $66 \%-96 \%$ of urogynecologists and urologists in the United Kingdom regarded preoperative UDS as essential in various clinical scenarios [19]. However, debate continues about whether preoperative UDS should be performed before incontinence surgery, because UDS involves invasive tests that require catheterization, such as cystometric analysis, urethral-pressure profiling, and leak-point pressure measurement.

Nager et al. [20] compared the outcomes between an officeevaluation-only group and a preoperative UDS group in a multicenter, non-inferiority, randomized trial (315 women per group). The office-evaluation-only group did not have significant inferiority in treatment success 1 year after surgery, and they also did not experience greater rates of other secondary outcomes, such as postoperative voiding dysfunction and impaired quality of life. van Leijsen et al. [21] also confirmed noninferiority in an office-evaluation-only group using the Urogenital Distress Inventory Urinary Incontinence Subscale at 1 year after surgery; however, the number of participants was small ( $\mathrm{n}=59$ women). Linder et al. [22] reported associations between preoperative urodynamic parameters and the risk of surgical sling release, leading to a postoperative voiding problem. None of the urodynamic parameters measured in this study, including peak flow rate, postvoiding residual volume, and detrusor pressure at maximal flow, showed a significant association with sling-release risk, although procedures involving the surgical-sling method were rare in this cohort.

In contrast, several studies have recommended preoperative UDS for incontinence surgery. Midurethral sling tension can be adjusted to be less obstructive if the Valsalva leak-point pressure is too low or if detrusor underactivity or intrinsic sphincter deficiency is diagnosed during a preoperative UDS [23]. In a study comparing the risk factors for failure between a retropubic tape group and a transobturator tape group, the odds ratios for mixed urinary incontinence and detrusor overactivity were 3.7 and 8.6, respectively, in the retropubic tape group compared with the transobturator tape group, showing that preoperative UDS could guide physicians in choosing the best surgical option [24]. Furthermore, preoperative UDS findings can help physicians and patients to predict postoperative urgency and urgency urinary incontinence. Lee et al. [25] reported that uro- 
dynamic parameters, such as detrusor overactivity, preoperative urgency symptom severity, and types of incontinence surgery, were significant risk factors for postoperative urgency or urgency urinary incontinence. These findings indicate that physicians can provide their patients more accurate counseling about potential voiding problems if they are guided by preoperative UDS data.

In our study, we could not investigate the efficacy of preoperative UDS as in previous studies, because most cases in which preoperative UDS was not performed in this cohort occurred in 2006 and early 2007. Thus, selection bias was likely to affect clinical outcome comparisons according to whether a preoperative UDS was performed. Instead, when stratified by performance of a preoperative UDS, the HRs for reoperation according to the surgeon's specialty varied based on whether a preoperative UDS was performed. While the reoperation rate was significantly lower in the procedures performed by urologists when a preoperative UDS was performed, there was no significant difference in the HRs for reoperation between the 2 groups when a preoperative UDS was not performed. This indicates that urologists consider adjusting midurethral tape tension or postoperative treatment such as anticholinergic therapy based on UDS findings, because they typically have more experience treating patients with voiding disorders beyond SUI according to UDS results.

Our study has several limitations. First, we did not investigate the distribution of different incontinence surgery types according to surgeon specialty. Furthermore, the surgical experience and learning curve of each surgeon also could not be considered in this population-based study. The reoperation and complication rates might have varied according to surgery type and the surgeon's experience. Thus, detection biases could have been a problem in this study. Second, this cohort did not include some important patient information, such as hysterectomy status, POP, and other comorbid diseases, with the exception of hypertension, diabetes, and dyslipidemia. These factors could affect the postoperative complication rate. Third, a matched case-control study estimating the value of preoperative UDS using a population-based cohort would be useful.

In conclusion, this population-based study showed that the postoperative outcomes of incontinence surgery depended on the surgeon's specialty and that the reoperation rate was significantly lower in procedures performed by urologists than in procedures performed by nonurologists. The reoperation rate according to the surgeon's specialty varied based on whether a preoperative UDS was performed. However, further research that accounts for the abovementioned limitations is needed to clarify the clinical risk factors and to improve the outcomes of incontinence surgery.

\section{AUTHOR CONTRIBUTION STATEMENT}

- Full access to all the data in the study and takes responsibility for the integrity of the data and the accuracy of the data analysis: $\mathrm{KDH}$

- Study concept and design: $J B C, S H H$

- Acquisition of data: $J B C, K D H$

- Analysis and interpretation of data: $K D H$

- Drafting of the manuscript: $J B C$

- Critical revision of the manuscript for important intellectual content: USH, SHH

- Statistical analysis: $\mathrm{KDH}$

- Obtained funding: $\mathrm{SHH}$

- Administrative, technical, or material support: $J B C, K D H$

- Study supervision: $\mathrm{USH}, \mathrm{SHH}$

\section{REFERENCES}

1. Haylen BT, de Ridder D, Freeman RM, Swift SE, Berghmans B, Lee J, et al. An International Urogynecological Association (IUGA)/International Continence Society (ICS) joint report on the terminology for female pelvic floor dysfunction. Neurourol Urodyn 2010;29:4-20.

2. Choi JB, Yoon BI, Han KD, Hong SH, Ha US. Urinary incontinence is associated with the development of peptic ulcers in adult women: Data from the KNHANES IV. Medicine (Baltimore) 2017; 96:e8266.

3. Hu TW, Wagner TH, Bentkover JD, Leblanc K, Zhou SZ, Hunt T. Costs of urinary incontinence and overactive bladder in the United States: a comparative study. Urology 2004;63:461-5.

4. Opara J, Czerwińska-Opara WE. The prevalence of stress urinary incontinence in women studying nursing and related quality of life. Prz Menopauzalny 2014;13:32-5.

5. Reynolds WS, Dmochowski RR, Penson DF. Epidemiology of stress urinary incontinence in women. Curr Urol Rep 2011;12:3706.

6. Choi H, Palmer MH, Park J. Meta-analysis of pelvic floor muscle training: randomized controlled trials in incontinent women. Nurs Res 2007;56:226-34.

7. Ulger Z, Cankurtaran M, Arýogul S. Weight loss for urinary incontinence in overweight and obese women. N Engl J Med 2009;360:2256-7. 
8. Malallah MA, Al-Shaiji TF. Pharmacological treatment of pure stress urinary incontinence: a narrative review. Int Urogynecol J 2015;26:477-85.

9. Wu JM, Gandhi MP, Shah AD, Shah JY, Fulton RG, Weidner AC. Trends in inpatient urinary incontinence surgery in the USA, 19982007. Int Urogynecol J 2011;22:1437-43.

10. Cho SY, Jeong SJ, Yeo JK, Kim SO, Jeong J, Choi JB, et al. Nationwide database of surgical treatment pattern for patients with stress urinary incontinence in Korea. Int Neurourol J 2014;18:91-4.

11. Kim JW, Moon du G, Shin JH, Bae JH, Lee JG, Oh MM. Predictors of voiding dysfunction after mid-urethral sling surgery for stress urinary incontinence. Int Neurourol J 2012;16:30-6.

12. Choi JB, Lee EJ, Han KD, Hong SH, Ha US. Estimating the impact of body mass index on bladder cancer risk: Stratification by smoking status. Sci Rep 2018;8:947.

13. James MB, Theofanides MC, Sui W, Onyeji I, Badalato GM, Chung DE. Sling procedures for the treatment of stress urinary incontinence: comparison of national practice patterns between urologists and gynecologists. J Urol 2017;198:1386-91.

14. Wu MP, Huang KH, Long CY, Huang KF, Yu KJ, Tang CH. The distribution of different surgical types for female stress urinary incontinence among patients' age, surgeons' specialties and hospital accreditations in Taiwan: a descriptive 10-year nationwide study. Int Urogynecol J Pelvic Floor Dysfunct 2008;19:1639-46.

15. Anger JT, Litwin MS, Wang Q, Pashos CL, Rodríguez LV. Variations in stress incontinence and prolapse management by surgeon specialty. J Urol 2007;178(4 Pt 1):1411-7.

16. Slopnick EA, Hijaz AK, Nguyen CT, Abouassaly R, Gonzalez CM, Mahajan ST, et al. National surgical trends and perioperative outcomes of midurethral sling placement for stress urinary incontinence. Urology 2017;99:57-61.

17. Elliott CS, Sokol ER. Might pelvic surgeons be unaware of their surgical failures? Patient reporting and perceptions after failed incontinence or pelvic organ prolapse surgery. Female Pelvic Med
Reconstr Surg 2015;21:298-300.

18. Spelzini F, Frigerio M, Regini C, Palmieri S, Manodoro S, Milani R. Learning curve for the single-incision suburethral sling procedure for female stress urinary incontinence. Int J Gynaecol Obstet 2017; 139:363-7.

19. Hilton P, Bryant A, Howel D, McColl E, Buckley BS, Lucas M, et al. Assessing professional equipoise and views about a future clinical trial of invasive urodynamics prior to surgery for stress urinary incontinence in women: a survey within a mixed methods feasibility study. Neurourol Urodyn 2012;31:1223-30.

20. Nager CW, Brubaker L, Litman HJ, Zyczynski HM, Varner RE, Amundsen $\mathrm{C}$, et al. A randomized trial of urodynamic testing before stress-incontinence surgery. N Engl J Med 2012;366:1987-97.

21. van Leijsen SA, Kluivers KB, Mol BW, Broekhuis SR, Milani AL, Bongers MY, et al. Can preoperative urodynamic investigation be omitted in women with stress urinary incontinence? A non-inferiority randomized controlled trial. Neurourol Urodyn 2012;31:1118-23.

22. Linder BJ, Trabuco EC, Gebhart JB, Klingele CJ, Occhino JA, Elliott DS, et al. Can urodynamic parameters predict sling revision for voiding dysfunction in women undergoing synthetic midurethral sling placement? Female Pelvic Med Reconstr Surg 2017 Nov 13 [Epub]. https://doi.org/10.1097/SPV.0000000000000521.

23. Sirls LT, Richter HE, Litman HJ, Kenton K, Lemack GE, Lukacz ES, et al. The effect of urodynamic testing on clinical diagnosis, treatment plan and outcomes in women undergoing stress urinary incontinence surgery. J Urol 2013;189:204-9.

24. Houwert RM, Venema PL, Aquarius AE, Bruinse HW, Roovers JP, Vervest HA. Risk factors for failure of retropubic and transobturator midurethral slings. Am J Obstet Gynecol 2009;201:202.e1-8.

25. Lee JK, Dwyer PL, Rosamilia A, Lim YN, Polyakov A, Stav K. Persistence of urgency and urge urinary incontinence in women with mixed urinary symptoms after midurethral slings: a multivariate analysis. BJOG 2011;118:798-805. 